\title{
UTILIZAÇÃO DAS FICHAS DE REGISTRO DE VACINAÇõES PELOS CENTROS DE SAÚdE NA DIVISÃO REGIONAL DE SAÚdE DE CAMPINAS - ESTADO DE SÃO PAULO, BRASIL *
}

\author{
William Moffitt HARRIS **
}

\begin{abstract}
\begin{tabular}{l|l} 
RSPU-B/224 \\
\hline
\end{tabular}
HARRIS, W. M. - Utilização das fichas de registro de vacinaçōes pelos centros de saúde na Divisão Regional de Saúde de Campinas, Estado de São Paulo, Brasil. Rev. Saúde públ., S. Paulo, 8:297-304, 1974.

RESUMo: Foi descrito um levantamento amostral comparativo analitico referente à coincidência de dados encontrados nas cadernetas de vacinações (amarelas) pertencentes a crianças e as fichas de registro (azuis) pertencentes aos arquivos dos Centros de Saude. Este levantamento foi feito em 16 municípios com mais de 30 mil habitantes da Região Administrativa de Campinas, Estado de São Paulo, que demonstrou haver em 788 cadernetas efetivamente examinadas, 2.307 anotaçöes divergentes referentes a vacinas. Apenas 386 $(48,9 \%)$ cadernetas amarelas coincidiam perfeitamente com as respectivas fichas azuis de registro. Tornou-se evidente que os arquivos de fichas de registro de vacinações dos Centros de Saúde não oferecem condições para uma eficiente avaliação da cobertura vacinal da população.
\end{abstract}

Unitermos: Centros de Saúde (Região de Campinas, Estado de São Paulo, Brasil)*; Vacinações *; Registro *; Imunizaçōes.

\section{N T R O D U C A O}

Teve por objetivo este estudo dimensionar pragmaticamente o desvio das normas que está havendo no registro das vacinações feitas ou referidas pelas famílias dos clientes dos Centros de Saúde da rede estatal, através de um levantamento casual em seus arquivos.

A caderneta de vacinações, de cor amarela (Fig. 1), que fica de posse da família da criança e sua correspondente ficha de registro, de cor azul ou acinzentada (Fig. 2), pertencente ao arquivo do Centro de Saúde, possuem em seu verso uma tabela para registro das doses apli- cadas das diversas vacinas e, de acordo com as normas, estes documentos deveriam conter exatamente as mesmas informações. Estas fichas foram criadas em 1968 pelo Grupo Executivo do Programa de Vacinações da Secretaria da Saúde Pública do Estado de São Paulo sob a presidência do então Secretário de Estado Professor Walter Leser.

O presente estudo foi conduzido em 16 municípios com mais de 30 mil habitantes da Divisão Regional de Saúde de Campinas (DRS-5), Estado de São Paulo, durante a campanha de vacinação múltipla

* Trabalho realizado no Serviço de Estudos e Programas (R5.Pr) da Divisão Regional de Saúde de Campinas (DRS.5) da Secretaria de Estado da Saúde de São Paulo

** Da Disciplina de Higiene da Crlanca do Departamento de Prática de Saúde Pública da Faculdade de Saúde Pública da USP - Av. Dr. Arnaldo, 715 - São Paulo, SP - Brasil 

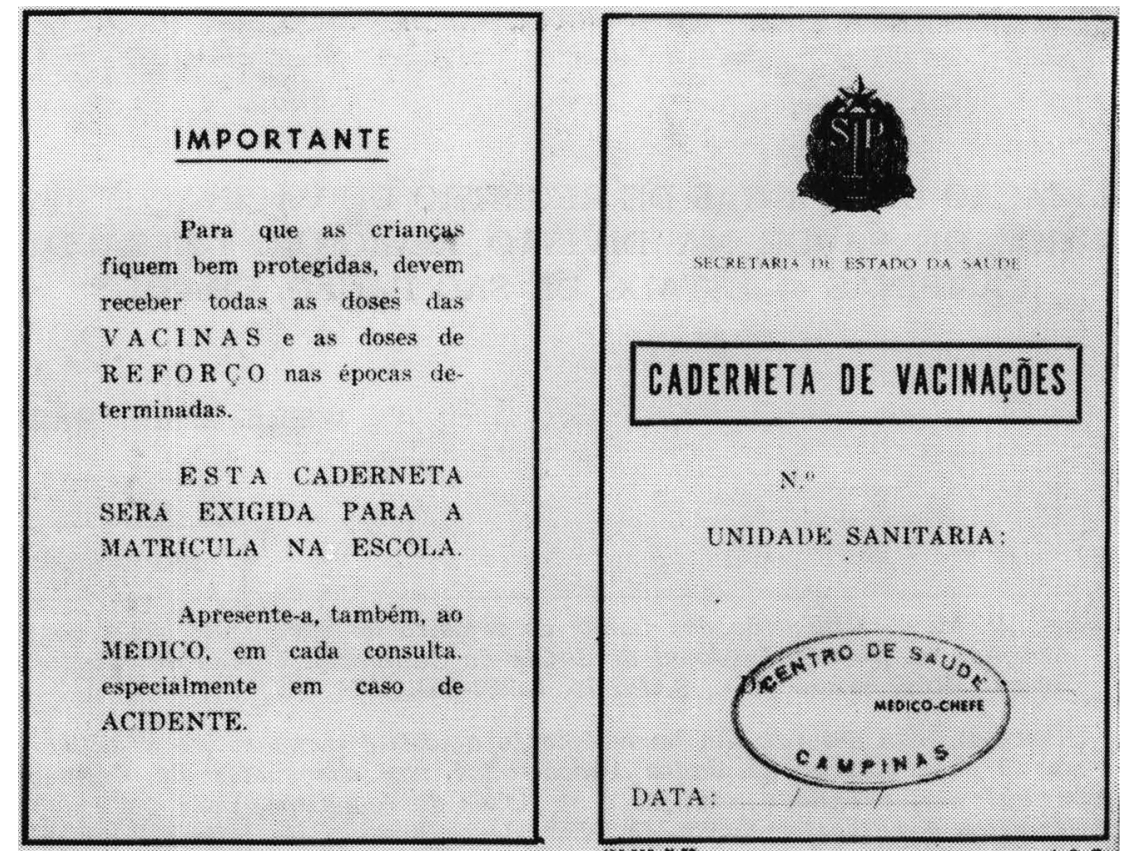

Fig. 1 - Fotografia do anverso da caderneta de vacinacões (amarela) instituída pela Secretarla de Estado da Saúde de São Paulo em 1968. Pertencente à criança.

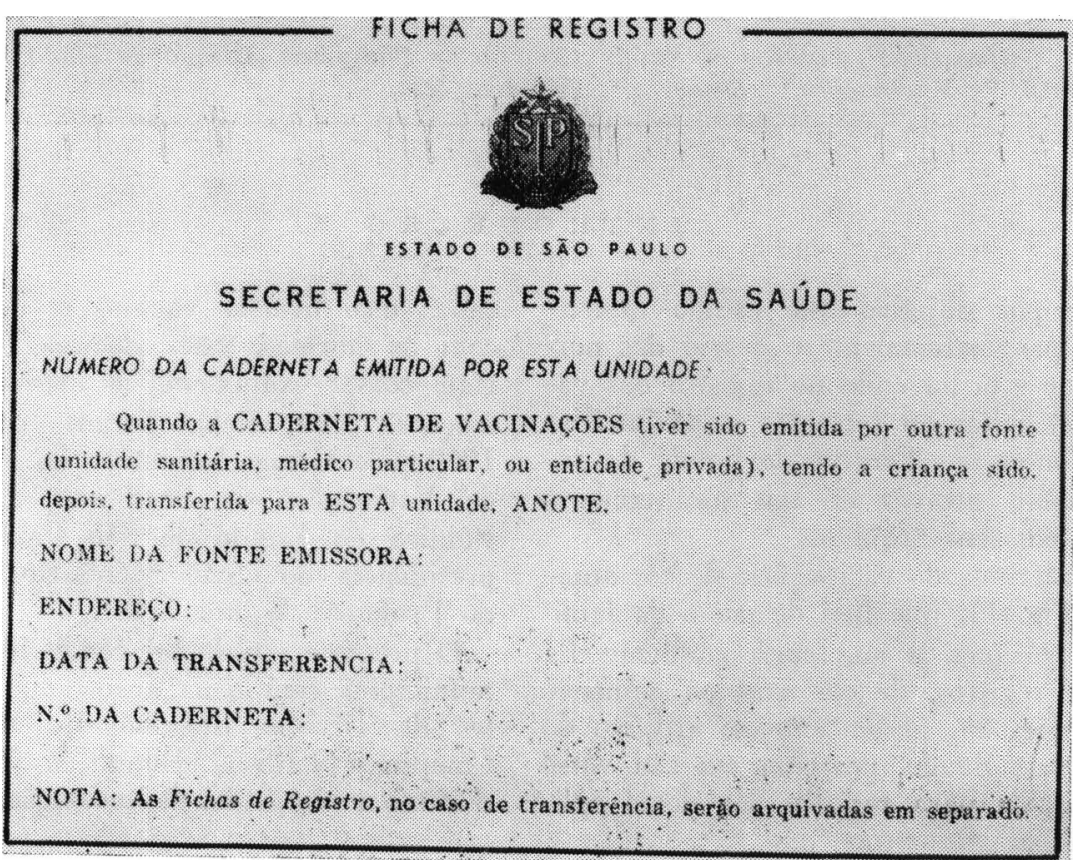

Fig. 2 -Fotografia do anverso da ficha de registro de vacinações (azul ou acinzentada) instituida pela Secretaria de Estado da Saúde de São Paulo em 1968. Pertencente ao arquivo do Centro de Saúde. 
HARRIS, W. M. - Utilização das fichas de registro de vacinacōes pelos centros de saúde nil Divisão Reglonal de Saúde de Campinas, Estado de São Paulo, Brasil. Rev. Saúde públ., S. Paulo, 8:297-304, 1974 .

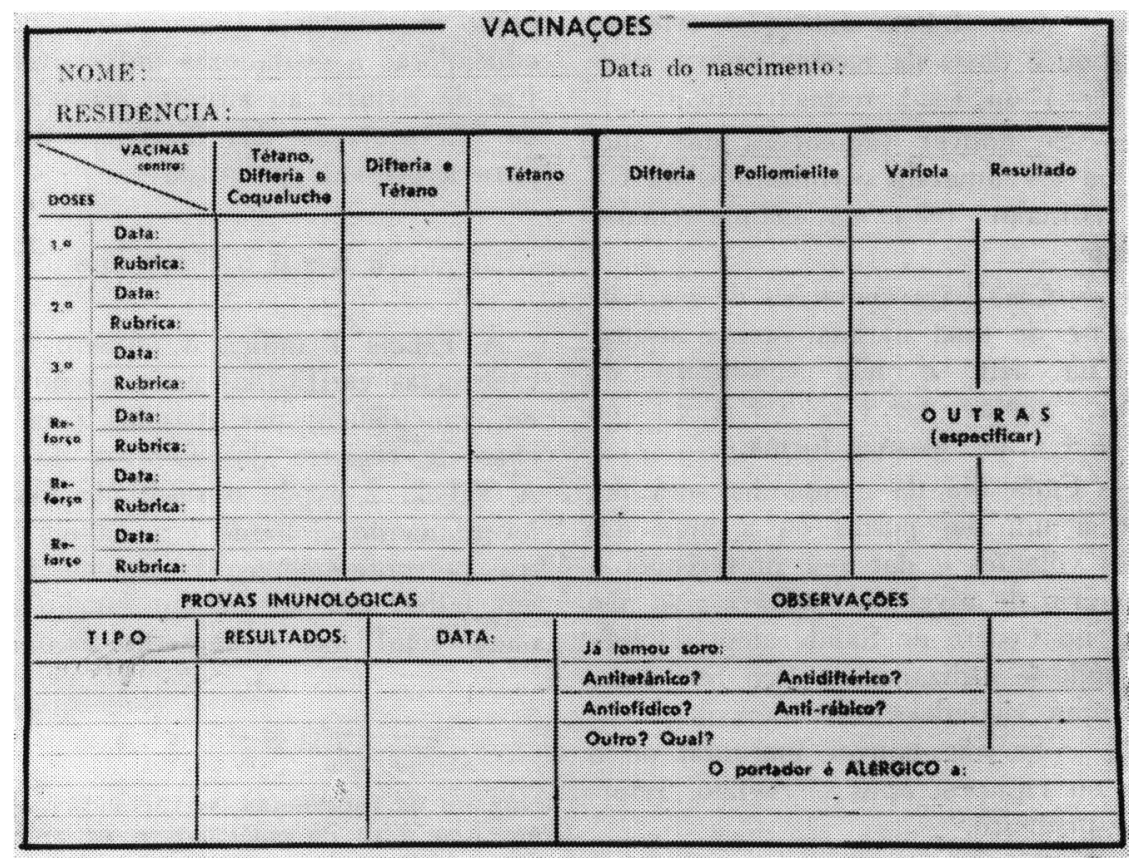

Fig. 3 - Fotografia do verso da caderneta de vacinaçōes e da ficha de registro de vacinaçōes referidas nas Fig. 1 e Fig. 2.

e simultânea, de 5 a 9 de novembro de 1973 - época em que o autor exercia o cargo de Diretor do Serviço de Estudos e Programas da DRS-5. Esta Regiāo Administrativa (uma das 11 do Estado) com 83 municípios contava na ocasião com uma população estimada aproximadamente de 2.300.000 habitantes, sendo que em 22 destes municípios havia mais de 30 mil. Embora o material tenha sido distribuído aos Centros de Saúde de todos estes 22 , seis não puderam apresentar condições operacionais satisfatórias para serem incluídos no presente estudo.

\section{MATERIAL E METODOS}

O levantamento foi executado aproveitando-se a oportunidade da campanha de vacinação múltipla e simultânea, durante a qual houve afluxo de considerável contingente de crianças menores de seis anos de idade e que constitui notoriamente o maior grupo portador de cadernetas de vacinaçōes.

Aos médicos chefes dos Centros de Saúde envolvidos na pesquisa foi enviada circular que continha as seguintes instruçōes:

"1 - Serão examinadas as cadernetas de vacinação (CV) amarelas e azuis de cada $10 .^{a}$ criança da fila que se forma no posto de vacinação.

1.1 - Se a criança sorteada não tiver caderneta passar para a seguinte, ou a após seguinte, e a partir desta contar mais 10.

1.2 - Se a criança tiver a CV amarela e não existir a $\mathrm{CV}$ azul no posto de vacinação, anotar na linha correspondente em todas as colunas o sinal $(-)$.

2 - Se houver vacinações anotadas na $\mathrm{CV}$ amarela e que não constam na $\mathrm{CV}$ azul, assinalar (-) na coluna correspondente. 
HARRIS, W. M. - Utilização das fichas de registro de vacinaç̃es pelos centros de saúde na Divisão Regional de Saúde de Campinas, Estado de Sāo Paulo, Brasill. Rev. Saúde públ., S. Paulo, 8:297-304, 1974

2.1 - Caso faltem anotações referentes a 2 ou 3 doses da mesma vacina, assinalar $(=)$ ou $(\equiv)$ respectivamente.

3 - Se houver vacinações não anotadas na CV amarela e constantes da $\mathrm{CV}$ azul, assinalar $(+)$ na coluna correspondente.

3.1 - Caso haja anotações de mais de uma dose de uma mesma vacina assinaladas na azul e não constantes da amarela, anotar $(++),(+++)$ ou $(+++)$ da mesma forma.

4 - Cada dia da campanha será dedicado a um dos postos ou a uma das equipes volantes e dar-se-á preferência a funcionário de nível universitário; se não houver no Centro de Saúde além do médico, pode-se utilizar para este fim específico uma visitadora universitária.

4.1 - Se não houver um total de 5 postos ou equipes, após o rodízio, retornar ao primeiro."

Estas instruções constavam do verso de uma tabela mimeografada que permitia anotar devidamente as informações solicitadas.

Em companhia da supervisora (Inspetora de Área Técnica Especializada) de Educação em Saúde de nível regional, o autor manteve contacto com os elementos de campo, quer na fase preparatória, quer na fase executiva da campanha a fim de explicar e observar o andamento desta e de outras pesquisas.

Consistia portanto a tarefa em simplesmente assinalar que informações havia a mais e que dados estavam faltando nas fichas de registro dos Centros de Saúde ao serem comparadas com as cadernetas de vacinações. Em muitos municípios estas cadernetas amarelas foram recolhidas para posterior comparação com as dos arquivos, face ao enorme movimento dos dias da campanha. Igual conduta foi tomada pelas equipes rurais que em sua maior parte não levaram consigo o fichário correspondente à zona a ser visitada em seu roteiro.

Preferiu-se utilizar pessoal de qualifi- cação universitária (enfermeiras, médicos, educadoras e visitadoras universitárias) a fim de reduzir ao mínimo possíveis erros de interpretação das diretrizes recebidas para a presente pesquisa.

\section{R E S U T A D O S}

A Tabela 1 indica o número total de cadernetas verificadas assim como o grau de coincidência das mesmas com as fichas de registro dos Centros de Saúde. A Tabela 2 expõe o resultado global do levantamento efetuado e a Tabela 3 mostra percentualmente $o$ dimensionamento das falhas por vacina em função do número total das cadernetas examinadas.

T A B E L A 1

Número de cadernetas de vacinaçōes verificadas e o grau de coincidência de seus dados com as fichas de registro dos Centros de Saúde, por município - Divisão Regional de Saúde de Campinas, Estado de São Paulo - 1973

\begin{tabular}{|c|c|c|c|}
\hline \multirow{3}{*}{ Municipio } & \multicolumn{3}{|c|}{ Cadernetas } \\
\hline & \multirow{2}{*}{$\begin{array}{l}\text { Número } \\
\text { total } \\
\text { exami- } \\
\text { nado }\end{array}$} & \multicolumn{2}{|c|}{$\begin{array}{c}\text { Colnciden- } \\
\text { tes }\end{array}$} \\
\hline & & N.o & $\%$ \\
\hline Americana & 54 & 2 & 3,7 \\
\hline Indaiatuba & 24 & 16 & 66,6 \\
\hline Amparo & 37 & 15 & 40,5 \\
\hline Itapira & 26 & 14 & 53,8 \\
\hline Bragança Paulista & 96 & 69 & 71,8 \\
\hline Atibaia & 28 & 0 & 0,0 \\
\hline Valinhos & 20 & 17 & 85,0 \\
\hline Jundiai & 192 & 40 & 20,8 \\
\hline Limeira & 49 & 23 & 46,9 \\
\hline Pirassununga & 22 & 0 & 0,0 \\
\hline Mogi-Mirim & 30 & 19 & 63,3 \\
\hline Mogi-Guaçú & 33 & 13 & 39,4 \\
\hline Piracicaba & 44 & 21 & 47,7 \\
\hline Santa Bárbara & 45 & 36 & 80,0 \\
\hline Rio Claro & 63 & 62 & 98,4 \\
\hline S. João da B. Vista & 50 & 39 & 78,0 \\
\hline Total & 788 & 386 & 48,9 \\
\hline
\end{tabular}


HARRIS, W. M. - Utilização das fichas de registro de vacinaçōes pelos centros de saúde na Divisão Regional de Saúde de Campinas, Estado de São Paulo, Brasil. Rev. Saúde públ., S. Paulo, 8:297-304, 1974.

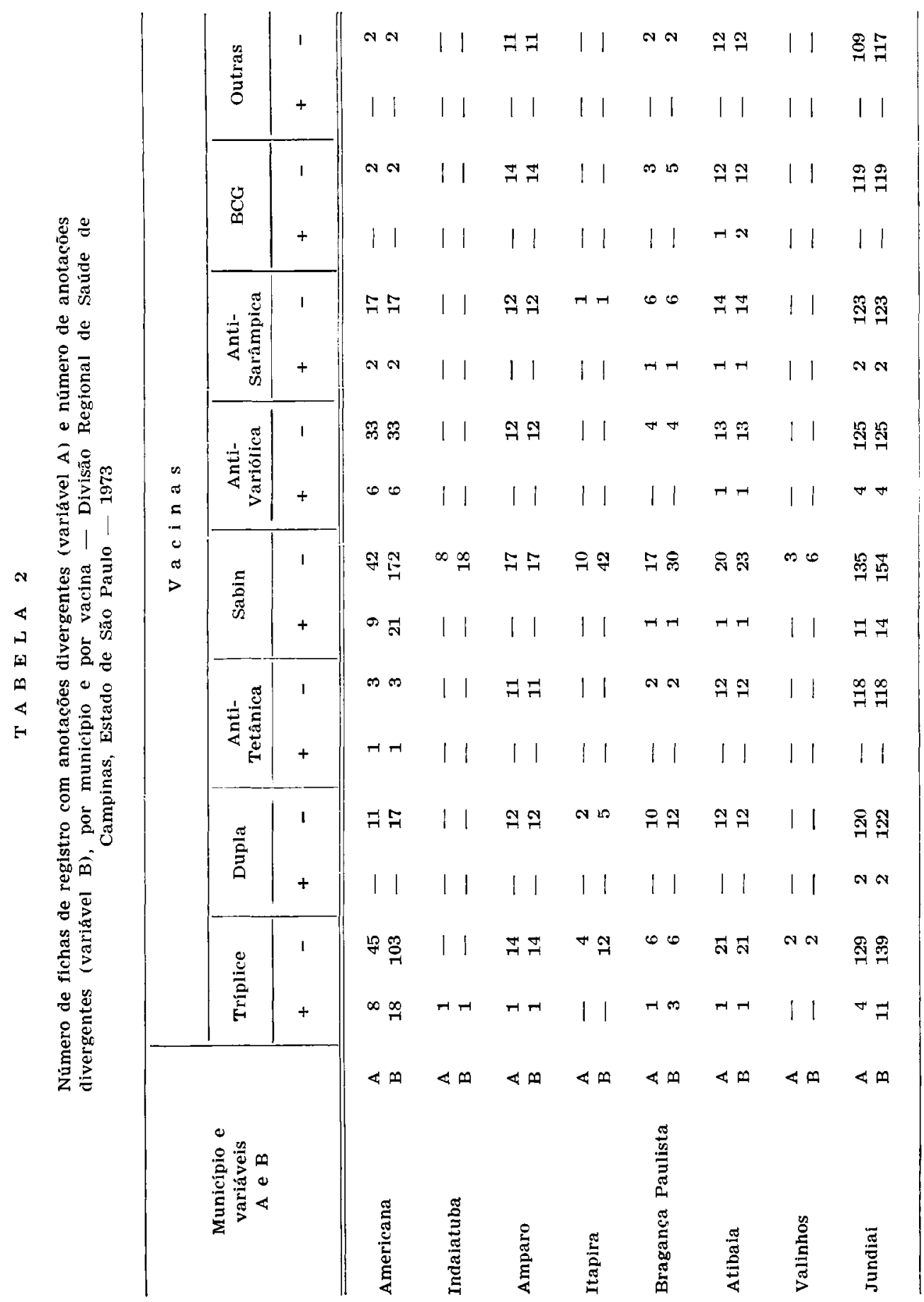


HARRIS, W. M. - Utilização das fichas de registro de vacinações pelos centros de saúde na Divisão Regional de Saúde de Campinas, Estado de São Paulo, Brasil. Rev. Saúde públ., S. Paulo, 8:297-304, 1974 .

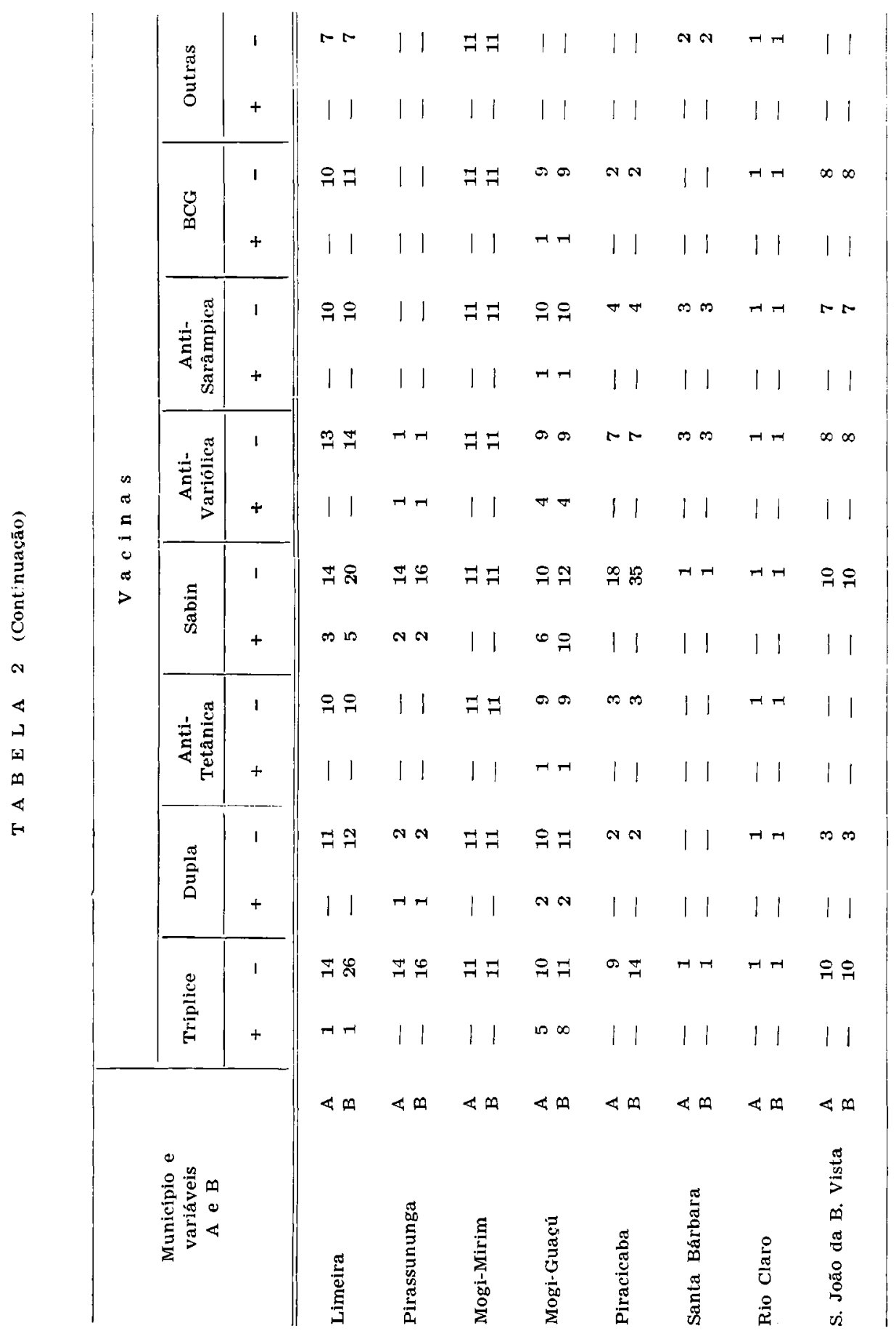


HARRIS, W. M. - Utilização das fichas de registro de vacinaçōes pelos centros de saúde na Divisão Regional de Saúde de Campinas, Estado de São Paulo, Brasil. Rev. Saúde pübl., S. Paulo, 8:297-304, 1974.

T A B E L A 3

Número de cadernetas de vacinações com anotações divergentes das fichas de registro em relação ao total de 788 examinadas e número de anotaçōes divergentes nas mesmas, por vacina, a menos (-) ou a mais $(+)$ ao serem comparadas com as fichas de registro dos Centros de Saúde - 16 municípios da Divisão Regional de Saúde de Campinas, Estado de São Paulo - 1973

\begin{tabular}{|c|c|c|c|c|}
\hline \multirow{2}{*}{ Vacinas } & & \multicolumn{2}{|c|}{ Cadernetas divergentes } & \multirow{2}{*}{$\begin{array}{l}\text { Número de } \\
\text { anotações } \\
\text { divergentes }\end{array}$} \\
\hline & & N. 0 & $\%$ & \\
\hline Triplice & $\overline{+}$ & $\begin{array}{r}22 \\
291\end{array}$ & $\begin{array}{r}2,8 \\
36,9\end{array}$ & $\begin{array}{r}44 \\
387\end{array}$ \\
\hline Dupla & $\bar{t}$ & $\begin{array}{r}5 \\
207\end{array}$ & $\begin{array}{r}0,6 \\
26,3\end{array}$ & $\begin{array}{r}5 \\
222\end{array}$ \\
\hline Anti-Tetânica & $\overline{+}$ & $\begin{array}{r}2 \\
180\end{array}$ & $\begin{array}{r}0,2 \\
22,8\end{array}$ & $\begin{array}{r}2 \\
180\end{array}$ \\
\hline Sabin & $\overline{+}$ & $\begin{array}{r}33 \\
332\end{array}$ & $\begin{array}{r}4,2 \\
42,1\end{array}$ & $\begin{array}{r}54 \\
568\end{array}$ \\
\hline Anti-Variólica & $\overline{+}$ & $\begin{array}{r}16 \\
240\end{array}$ & $\begin{array}{r}2,0 \\
30,5\end{array}$ & $\begin{array}{r}16 \\
241\end{array}$ \\
\hline Anti-Sarâmpica & $\overline{+}$ & $\begin{array}{r}7 \\
219\end{array}$ & $\begin{array}{r}0,9 \\
27,8\end{array}$ & $\begin{array}{r}7 \\
219\end{array}$ \\
\hline $\mathrm{BCG}$ & $\overline{+}$ & $\begin{array}{r}2 \\
191\end{array}$ & $\begin{array}{r}0,2 \\
24,2\end{array}$ & $\begin{array}{r}3 \\
194\end{array}$ \\
\hline Outras vacinas & $\overline{+}$ & $\begin{array}{r}0 \\
157\end{array}$ & $\begin{array}{r}0,0 \\
19,9\end{array}$ & $\begin{array}{r}0 \\
165\end{array}$ \\
\hline$T \circ t$ a $l$ & & - & - & 2.307 \\
\hline
\end{tabular}

Assim, por exemplo, verificou-se: que $23,0 \%$ das cadernetas examinadas continham anotações divergentes relativas à vacinação antitetânica; que um total de 332 cadernetas dos 788 verificados (e portanto $42,1 \%$ do total) continham 568 anotações divergentes a mais relativas à vacinação antipoliomielítica (Sabin); e que havia $7(0,9 \%)$ cadernetas azuis com registro de vacinações contra sarampo efetuadas nos Centros de Saúde, sem que isto figurasse nas respectivas cadernetas de vacinações das crianças.

\section{DISCUSSAO E CONCLUSOES}

Certamente as informações registradas nos fichários e arquivos de unidades sanitárias deveriam ser utilizadas como prérequisitos básicos e essenciais para um planejamento bem sucedido no setor saúde. O cálculo da cobertura vacinal de determinados grupos da população susceptível a certas moléstias permite equacionar prioridades, dimensionar a utilização de recursos e estruturar programaçōes especiais para interromper cadeias epidemiológicas. 
HARRIS, W. M. - Utilização das fichas de registro de vacinaçóes pelos centros de saúde na Divisão Regional de Saúde de Campinas, Estado de São Paulo, Brasil. Rev. Saúde públ., S. Paulo, 8:297-304, 1974.

Este trabalho pretendeu contribuir para o melhor conhecimento da realidade ao nível local quanto à validade destes registros, servindo de alerta aos planejadores de saúde, apontando como prováveis causas destas discrepâncias:

1 - falta de adequado treinamento de pessoal de nível local;

2 - falta de número necessário de funcionários para a completa execução das tarefas de registro. por ocasião da extraordinária demanda aos Centros de Saúde nas campanhas de vacinação;

3 - falta de esclarecimento da população em geral quanto à importância de retornar sempre aos mesmos locais para as vacinaçōes subsequientes das crianças, a fim de que não haja possibilidade de estarem anotadas em diversas fichas de registro as vacinações de uma mesma criança.

A G R A D E C I M E N T O S

A todos os servidores da Divisão Regional de Saúde de Campinas (DRS-5) que participaram da coleta e tabulação dos dados do presente trabalho, principalmente aos colegas da embrionária Seção de Planejamento do Serviço de Estudos e Programas.

RSPU-B $/ 224$

HARRIS, W. M. - [The use of the vaccination record cards by the local health centers of the Campinas Regional Health Division of the State of S. Paulo, Brazil.J Rev. Saúde públ., S. Paulo, 8:297-304, 1974.

Summary: A comparative analytical sample survey regarding the coincidence of data found in Vaccination Record Cards (yellow ones) belonging to children and those (blue ones) belonging to the local Health Units' archives conducted in 16 connties with populations over thirty thousand among those pertaining to the Campinas Administrative Region 183 counties with a total population of 2.3 million) of the State of S. Paulo, Brazil, was here described showing that in 788 cards effectively examined there were 2,307 diverging vaccine informations. Only $386(48.9 \%)$ Yellow Cards coincided perfectly with their corresponding Blue Cards. It became quite obvious that the vaccination archives of the local Health Units do not yield conditions for an efficient vaccinal coverage evaluation of the population.

UNITERMS: Health Centers (Region of Campinas, State of S. Paulo, Brazil) *; Vaccinations*; Vaccination Records*; Immunization. 